\title{
La traduction et le fascisme : quelques réflexions à partir des théories de Croce et Gentile*
}

\author{
Viviana AGOSTINI-OUAFI \\ Université de Caen Normandie \\ France
}

\begin{abstract}
Résumé : L’intervention évoque la vitalité du marché des traductions dans l'Italie fasciste, malgré la censure touchant notamment Gramsci traducteur et théoricien de la traduction, et étudie les théories de l'entre-deux-guerres en partant de la polémique de 1920 entre les philosophes idéalistes Croce et Gentile. L'antifasciste Croce nie la possibilité de la traduction au nom d'une idée de l'art aristocratique et romantique, poussant ainsi les critiques de son camp comme Debenedetti à emprunter des voies différentes. La conception de l'art chez Gentile, qui prétend incarner l'intellectuel fasciste, est en contradiction avec sa conception du pouvoir : déconstruction subjective et autoritarisme étatique, liberté interprétative et violence idéologique cohabitent au point que ses réflexions traductologiques se rapprochent absurdement de celles de Benjamin.
\end{abstract}

Mots-clés : fascisme, idéalisme, traduction, Gramsci, Gentile, Croce, Debenedetti.

\begin{abstract}
This paper focuses on the vitality of the translation market in fascist Italy, despite the censorship affecting primarily Gramsci as a translator and theorist of translation, and, starting from the 1920 polemic between the idealist philosophers Croce and Gentile, it studies the theories emerging between the two world wars. The anti-fascist Croce denies the possibility of translating in the name of an aristocratic and romantic idea of art, and thus pushes critics in his camp, such as Debenedetti, to resort to different paths. Although Gentile claims to embody the fascist intellectual, his view on art is in contradiction with his view on power: subjective deconstruction and state authoritarianism, interpretive freedom and ideological violence coexist to the point where his reflections on translation get absurdly close to Benjamin's.
\end{abstract}

Key words: fascism, idealism, translation, Gramsci, Gentile, Croce, Debenedetti.

Dans les années 1920, la réflexion sur la traduction connaît en Italie un essor remarquable qui n'est pas toujours assez mis en valeur : son impact sur la culture de l'entre-deux-guerres et plus généralement du XXesiècle a été conditionné par les choix politiques que les différents protagonistes ont faits face au fascisme. En effet, nous allons

*Nous remercions les Presses universitaires de Caen pour nous avoir permis de republier cette étude qui a d'abord paru, avec ce même titre, dans la revue Transalpina, $\mathrm{n}^{\circ} 1$ : Fascisme et critique littéraire. Les hommes, les idées, les institutions, Christian Del Vento et Xavier Tabet (éd.), 2010,15-32. 
considérer ici le débat sur la traduction de 1920 entre Giovanni Gentile et Benedetto Croce $^{1}$, deux philosophes idéalistes qui ont travaillé ensemble pendant de longues années mais qui rédigent et signent respectivement le manifeste des intellectuels fascistes (21 avril 1925) et le manifeste des intellectuels antifascistes (1er mai 1925) ${ }^{2}$. C'est la Marche sur Rome qui conduira à leur rupture définitive. Selon József Nagy, malgré leurs différences idéologiques notables, avant cette rupture ils partagent sur le plan politique au moins deux présupposés, à savoir que le fascisme est la continuation du Risorgimento et qu'il constitue une période de transition vers le vrai libéralisme (2004, 81). Leurs conceptions de la traduction sont en revanche déjà très éloignées en 1920, même si les deux ont une approche herméneutique. Selon János Kelemen, leur pensée constitue l'un des moments essentiels de la formation de la philosophie herméneutique moderne mais leurs théories sont radicalement opposées : chez Croce l'interprétation est conçue comme "reproduction », chez Gentile comme «traduction ${ }^{3}$ (80). Pour le premier, dès 1902, la signification de l'œuvre d'art est à rechercher dans une seule intuition (ou image interne) : chaque œuvre a une unique interprétation ; par contre l'approche théorique de Gentile est, dès le début, subjectiviste et relativiste. D’après Nagy, cette conception crocienne rigide de l'œuvre d'art s'assouplit vers la fin des années 1930 lorsqu'il rédige La Poesia : l'identification de l'intuition originale devient alors pour Croce un but idéal impossible à atteindre. Cette conclusion correspond aux thèses prônées depuis toujours par Gentile portant sur l'infinité des possibilités interprétatives :

Ironizzando, si potrebbe dire che, per rimanere liberale nel corso dei tempi, Croce ha dovuto modificare in modo significativo la propria teoria d'interpretazione, mentre Gentile, per rimanere fascista, non ha dovuto modificar[la] affatto (80).

1 B. Croce, «Possibilità e impossibilità delle traduzioni » (1920), in Conversazioni critiche, vol. IV, Bari, Laterza, 1951, 308-309. Ce texte est un commentaire des thèses que Gentile expose dans « Il torto e il diritto delle traduzioni ", in Frammenti di estetica e letteratura, Lanciano, Carabba, 1920, 369-375. Au sujet de cette polémique, cf. R. Raggiunti, «Il concetto del tradurre nel pensiero di Giovanni Gentile », Giornale critico di filosofia italiana, s. III, XXIX, $\mathrm{n}^{\circ}$ 4, 1950, 443-452 ; N. Mátyus, " Il dibattito di Croce e Gentile sul problema della traduzione ", in Benedetto Croce 50 anni dopo, K. Fontanini, J. Kelemen, J. Takács (dir.), Budapest, Aquincum Kiadó, 2004, 443-449.

2 Leurs rapports, caractérisés dès 1896 par une étroite collaboration, sont marqués à partir de 1913 par une rupture philosophique officielle; cf. G. Galasso, Croce e lo spirito del suo tempo, Milan, Il Saggiatore, 1990, 103, 165 et passim. Leur dernier échange de lettres date de fin 1924 (cf. 1990, 312313).

3 Nagy commente l'œuvre de J. Kelemen, Az olasz hermeneutika Crocétól Ecóig [Ermeneutica italiana da Croce ad Eco], Budapest, Kávé K., 1998. 
Pour que l'opposition entre fascisme et antifascisme chez Croce et Gentile soit davantage illustrée dans toute son ambiguïté idéologique, rappelons cette définition du libéralisme donnée par Croce et que Nagy considère comme emblématique :

"Per liberalismo, che è nato e intrinsicamente rimane antiegualitario, la libertà [...] è la via per produrre e promuovere, non la democrazia, ma l'aristocrazia [...] aperta » che da una parte è ferma « a respingere il volgo », dall'altra parte è sempre "pronta ad accogliere chi a lei s'innalza $» 4(85)$.

Quant à la philosophie de Gentile, d'après Nicola Abbagnano, elle doit être comprise et jugée indépendamment du fascisme, d'où elle n’a tiré ni son origine ni son inspiration : le fascisme, pour Abbagnano, à ses débuts, n'avait aucune doctrine précise, sauf un nationalisme générique et intolérant ; mais Nagy rappelle que Gentile a adhéré sans aucun problème aux lectures totalitaires de l'œuvre de Hegel et de celle de Rousseau et interprété le premier de façon antimarxiste (Abbagnano 2006, 170 ; Nagy 2004, 81). Outre les positions de Gentile et Croce, pour brosser le panorama complexe des théories traductologiques de l'entre-deux-guerres, nous analyserons brièvement les réflexions sur ce sujet écrites en prison par le fondateur du Parti communiste italien Antonio Gramsci ainsi que sa démarche de traducteur5. Pour mettre en évidence certains préjugés, nous comparerons les réflexions de Gentile à celles de Walter Benjamin, similaires et proches dans le temps. Pour finir, nous prendrons en compte les remarques sur la traduction du critique littéraire Giacomo Debenedetti car ce dernier représente bien la nouvelle génération antifasciste qui dans les années 1920 doit se frayer un chemin entre Croce et Gentile ${ }^{6}$. Ces quatre intellectuels italiens nous permettront de situer les problèmes d'ordre linguistique et esthétique liés aux questions traductologiques dans un discours culturel et idéologique plus large pour tenter de saisir les failles, les points forts et les contradictions d'une relation entre histoire et culture qui n'est jamais banale ni acquise et souvent même pas cohérente.

On peut déjà balayer certains préjugés qui pourraient paraitre logiques : la censure fasciste et sa haine de l'étranger (xénophobie, anti-

4 Nagy cite ici B. Croce, «Elementi di politica », in Saggi filosofici, vol. VI, Etica e politica. Aggiuntovi il "Contributo alla critica di me stesso », Bari, Laterza, 1931, 213. C'est Croce qui souligne.

5 Cf. L. Borghese, "Tia Alene in bicicletta. Gramsci traduttore dal tedesco e teorico della traduzione », Belfagor, XXXVI, 1981 / 6, 635-665.

6 Cf. V. Agostini-Ouafi, Poetiche della traduzione. Proust e Debenedetti, M.T. Giaveri (intr.), Modène, Mucchi (Strumenti. Seconda serie : Opuscoli di teoria, storia e pratiche della traduzione, sous la direction d'A. Lavieri), 2010, notamment 30-52. 
européisme) ont-elles eu des répercussions importantes sur le marché éditorial de l'époque ? En s'appuyant sur l'histoire quantitative du livre et l'approche sociologique des phénomènes culturels, des études sur les traductions en Italie dans l'entre-deux-guerres ont démontré que la censure a été très tardivement efficace, qu'elle a favorisé certaines productions étrangères au détriment d'autres sans jamais bloquer la vitalité du marché éditorial7. Dans un ouvrage exemplaire, paru récemment, Valerio Ferme nous démontre par exemple que dans le domaine des traductions littéraires (et philosophiques) de l'angloaméricain vers l'italien, on passe de 17 auteurs et 52 livres traduits dans la période 1921-1925 à 29 auteurs et 165 livres entre 1926 et 1930, c'està-dire après l'instauration effective de la dictature ; le transfert traductif allant de l'Amérique du Nord vers l'Italie s'accélère entre 1931 et 1935, en plein renforcement du régime, puisqu'on atteint 49 auteurs et 228 livres traduits ; en revanche le phénomène va décroître légèrement entre 1936 et 1940 (48 auteurs et 208 livres), après l'invasion de l'Éthiopie et les sanctions de la Société des Nations envers l'Italie fasciste (2002, 223-228). Ces sanctions économiques entraînent en effet une vague d'anglophobie dans la péninsule (mais la langue française aussi en pâtit) ${ }^{8}$. Par ailleurs, Gramsci lui-même rappelle que dans les années 1920, les feuilletons et les romans historiques français ont envahi le marché italien de la paralittérature et que ces traductions, étant très mal payées, sont aussi de très mauvaise qualité : d'où une production nationale-populaire française remplaçant une littérature secondaire italienne lacunaire ${ }^{9}$; la maison d'édition Mondadori lance quant à elle en 1930 une collection populaire à succès consacrée aux

\footnotetext{
7 Cf. J.-P. Viallet, "Statistiques et histoire des relations culturelles francoitaliennes : l'exemple des traductions (1932-1939) », in Il vincolo culturale tra Italia e Francia negli anni trenta e quaranta, J.-B. Duroselle, E. Serra (dir.), Milan, Franco Angeli, 1986, 246-294; P. Vitiello, «Lettura del traduttore, lettura della traduzione : La Certosa di Parma e il progetto della "Biblioteca romantica Mondadori” », Esperienze letterarie, XV, 1990 / 3, 43-77.

8 Cf. G. Rando, "Anglicismi nel Dizionario moderno dalla quarta alla decima edizione », Lingua Nostra, XXX, 1969 / 4, 107-112.

9 Cf. A. Gramsci, Letteratura e vita nazionale, Rome, Editori Riuniti, 1977, 1718, 77, 99-101 et passim. Selon Gramsci, ces traductions contribuent à la francisation de la culture "nationale-populaire» italienne: "Questi libri stranieri, tradotti, sono letti e ricercati e conoscono spesso grandi successi. Tutto ciò significa che tutta la "classe colta", con la sua attività intellettuale, è staccata dal popolo-nazione, non perché il popolo-nazione non abbia dimostrato e non dimostri di interessarsi a questa attività in tutti i suoi gradi, dai più infimi (romanzacci d'appendice) ai più elevati, tanto vero che ricerca $\mathrm{i}$ libri stranieri in proposito, ma perché l'elemento intellettuale indigeno è più straniero degli stranieri di fronte al popolo-nazione » (ibid., 129). Pour les traductions du français, cf. L. Erba, " Mezzo secolo di traduzioni dal francese in Italia (1900-1950) », in Huysmans e la liturgia, Bari, Adriatica Editrice, 1971, 89-114.
} 
littératures étrangères traduites, "La Biblioteca romantica », sous la direction du critique littéraire et écrivain G.A. Borgese ${ }^{10}$. De même, en 1937, en pleine hystérie xénophobe, rien n'empêche que l'œuvre d'un auteur juif, homosexuel et « décadent » comme Marcel Proust, devenu symbole d'antifascisme, puisse être traduite dans une revue de Bologne au titre significatif : L'Italiano ${ }^{11}$. Autant de failles et de contradictions anecdotiques dans la gestion de la culture de l'époque, dévoilant peutêtre la confusion idéologique et l'hétérogénéité culturelle qui marquent la mouvance fasciste au moins jusqu'aux lois raciales.

La censure du régime s'exerce en revanche de façon évidente lorsque les intellectuels antifascistes sont obligés de s'expatrier ou sont emprisonnés. La relation entre traduction et fascisme est de ce point de vue claire si l'on considère l'œuvre et le destin tragique d'Antonio Gramsci. Ce dernier mourra en prison dans les années 1930 et sa réflexion traductologique ne sera étudiée en Italie que dans la seconde moitié du XXe siècle ${ }^{12}$. Sa pensée peut être résumée dans cette formule, tirée d'un chapitre des cahiers de prison portant justement sur la traductibilité des langages scientifiques et philosophiques: "Due strutture fondamentalmente simili hanno superstrutture "equivalenti" e reciprocamente traducibili, qualunque sia il linguaggio particolare nazionale ${ }^{13}$. Son approche sociologique de la traduction est par ailleurs en accord avec sa conception de la littérature et avec sa pratique de traducteur. En effet, il traduit en prison plusieurs ouvrages de

10 Cf. P. Vitiello, "Lettura del traduttore... », 52 : Borgese affirme avoir pu choisir en toute liberté cinquante chefs-d'œuvre étrangers, selon les goûts esthétiques de l'auteur, la célébrité du roman, les difficultés de traduire le rythme, l'affinité entre le traducteur et l'œuvre...

${ }_{11}$ M. Proust, «Soggiorno veneziano », L'Italiano, XII, 1937 / 5, 49-52. Sur l'accueil de Proust dans l'entre-deux-guerres, cf. G. Bosetti, « Signification socioculturelle et sociopolitique du proustisme en Italie », in Proust en Italie, V. Agostini-Ouafi (dir.), Transalpina, $\mathrm{n}^{\circ}$ 7, 2004, 27-40.

${ }^{12}$ Selon Lucia Borghese, on trouve une première mise en valeur de son idée de traduction dans l'œuvre de L. Paggi, Gramsci e il moderno principe, vol. I, Nella crisi del socialismo, Rome, Editori Riuniti, 1970 ; et les cahiers des traductions, qu'il rédige entre 1929 et 1931, sont encore inédits en 1981, mis à part une anthologie de 1980 où ils font l'objet, d'après elle, d'une " grottesca manipolazione » (« Tia Alene in bicicletta... », 636, note 2 ; 637).

13 A. Gramsci, Il materialismo storico e la filosofia di Benedetto Croce, Rome, Editori Riuniti, 1977, 83-84. Cf. ibid., 80: " osì due culture nazionali, espressioni di civiltà fondamentalmente simili, credono di essere diverse, opposte, antagonistiche, una superiore all'altra, perché impiegano linguaggi di tradizione diversa [...]: linguaggio politico-giuridico in Francia, filosofico, dottrinario, teorico in Germania. Per lo storico, in realtà, queste civiltà sono traducibili reciprocamente, riducibili l'una all'altra. Questa traducibilità non è "perfetta" certamente, in tutti i particolari, anche importanti (ma quale lingua è esattamente traducibile in un'altra? quale singola parola è traducibile esattamente in un'altra lingua ?) ma lo è nel "fondo" essenziale ». 
linguistique et de littérature allemandes, dont des contes pour enfants, et il prône dans cette activité les mêmes principes caractérisant sa théorie de la littérature : la démarche de Gramsci traducteur des frères Grimm est par exemple parfaitement cohérente avec son souci de créer une culture italienne nationale-populaire qui soit adaptée à son public méditerranéen et capable d'exercer sur lui une action éducative (il remplace dans un conte le drapeau rouge symbole de mort par un drapeau noir évoquant ainsi sa situation politique, ailleurs il adapte la toponymie nordique à celle du paysage sarde et il remplace les lièvres que chasse le renard par les poules plus familières, comme il opte toujours pour la laïcisation de toute expression véhiculant une conception religieuse du monde) (Borghese 1981, 653-656). On peut donc regretter que la réflexion de Gramsci sur le marché éditorial des traductions, analysé selon une conception matérialiste de l'histoire littéraire, ait fait défaut dans l'entre-deux-guerres à la culture italienne. Quant à une approche sociologique et systémique de la traduction semblable à la sienne, il faudra attendre qu'Itamar Even-Zohar, se réclamant des formalistes russes, esquisse au début des années 1970 sa théorie du polysystème ${ }^{14}$. Cette théorie, dite de l'école de Tel-Aviv, commencera à être connue en Italie à la fin des années 1980 : une mise en valeur moins tardive de la réflexion gramscienne sur la traduction nous aurait permis, peut-être, d'être davantage réceptifs.

Censure et répression ayant marginalisé très tôt la pensée et l'œuvre de Gramsci, le débat traductologique italien de l'entre-deuxguerres aurait dû tourner autour des deux philosophes idéalistes qui incarnent les rivages politiques opposés du fascisme et de l'antifascisme, Gentile et Croce. Nous savons par exemple que le jeune étudiant Debenedetti suivait attentivement les débats entre ces deux philosophes pour déterminer laquelle de ces deux conceptions en lice était la plus à même "d'illuminer l'idée de l'art » $(1969,20)$. Dans ce « grand duel » entre Croce et Gentile, Debenedetti se considère comme un disciple de Croce, quoique insatisfait de ses théories esthétiques. Comme il l'affirme en 1949, la conception de l'œuvre artistique que prône Gentile dans sa Filosofia dell'Arte (ouvrage paru en 1931) ne suscite chez lui aucun élan de sympathie, ne répond à aucune de ses attentes (24). De fait, Croce semble dominer par ses théories esthétiques les milieux antifascistes de l'époque; Giuseppe Galasso

14 I. Even-Zohar, «Le relazioni tra sistema primario e sistema secondario all'interno del polisistema letterario », Strumenti critici, IX, nº 26, 1975, 71-79. Sur la théorie du polysystème et les analyses de Gramsci, cf. notre étude : "Réception et traduction dans les échanges culturels contemporains entre la France et l'Italie ", in Les écrivains italiens et leurs traducteurs français, M. Colin, M.-J. Tramuta, V. Agostini-Ouafi (dir.), Caen, Presses universitaires de Caen, 1996, 76-79. Cf. aussi les liens entre J. Tynianov, « De l'évolution littéraire » (1927), in Théorie de la littérature, T. Todorov (trad. et éd.), Paris, Seuil, 1965, 120-127, et A. Gramsci, Letteratura e vita nazionale, 5 . 
affirme à ce sujet que son influence a été plus profonde et féconde en Italie sur les hommes de lettres et les historiens que sur les philosophes (1990, 305, 307). Ceci est confirmé aussi par Gianni Vattimo ; en 1954, Croce est considéré comme un maitre de l'esthétique, quoique trop littéraire et idéaliste; sa figure est importante pour l'histoire de la culture ou des idées et pour la critique littéraire, mais le vrai philosophe est Gentile, « un personaggio reso tragicamente grande dalla sua fine e, prima, dalla sua mal collocata fede politica, certo però infinitamente più geniale e profondo del suo rivale napoletano » $(2004,111)^{15}$. Dans les années 1950, on ne lit pas beaucoup Croce ni Gentile, on reproche au premier d'avoir éloigné la culture italienne des courants les plus actuels de la pensée, au deuxième sa compromission avec le fascisme : « un dato che allora contava ancora molto e non era stato consumato dai revisionismi successivi »16 (111).

Pour les intellectuels antifascistes des années 1920, une réflexion constructive sur la traduction ne peut se faire que malgré Croce car celui-ci, depuis le début du siècle et de façon nette encore en 1920, prône l'impossibilité de la traduction. En 1902, il a déclaré dans son Estetica que toute traduction déprécie, voire abîme, l'expression originale ou bien crée une nouvelle expression dont le contenu est nécessairement différent ${ }^{17}(1950,76)$. En 1920, dans sa polémique avec Gentile, il réitère ce jugement en soutenant que la seule lecture capable d'éveiller et de récréer l'émotion authentique que l'œuvre littéraire produit chez son premier lecteur est celle qui est faite... dans la langue originale (309). Comme le souligne Raimondo Filippi, il faut placer la thèse de Croce concernant l'impossibilité des traductions dans le contexte de ses théories philosophiques (1992, 33). En effet, selon son esthétique, intuition et expression poétiques sont indivisibles, si bien que la traduction est impossible par principe. Selon Filippi, le point de vue de Croce a même par la suite fortement conditionné «les approches des théoriciens [de la traduction] italiens en leur communiquant une sorte de préjugé négatif sur la valeur, du moins théorique, des traductions » (33). Roberto Fertonani partage cet avis : il souligne de façon catégorique que, du fait de Croce, le discrédit de la traduction s'est répandu non seulement dans les institutions universitaires mais aussi dans la mentalité commune (1957, 250).

15 Il meurt en effet à Florence le 14 avril 1944, exécuté froidement par des résistants florentins, devant la grille de son jardin.

16 M.F. Sciacca affirme par exemple en 1943 que l'autorité de Croce dans l'entre-deux-guerres n'est liée désormais qu'à son choix politique (cf. G. Galasso, Croce e lo spirito..., 496, note 48).

17 Cf. à ce propos G. Steiner, Après Babel (1975), Paris, Albin Michel, 1978, 230 ; G. Mounin, Les belles infidèles, Paris, Cahiers du Sud, 1955, p. 24-25; R. Filippi, "La théorie de la traduction en Italie au XXe siècle (1900-1960) », Équivalences : revue de l'Institut supérieur des traducteurs et interprètes de Bruxelles, nº 21, 1992 / 1-2, notamment 32-40. 
L'image que l'élite intellectuelle se fait de la traduction littéraire dans l'entre-deux-guerres n'est pas du tout positive: Pippo Vitiello nous donne plusieurs témoignages représentatifs de la surestimation de la lecture du texte original au détriment des traductions. Non seulement, à l'instar de Croce, l'élite italienne préfère la lecture des romans étrangers en langue originale, mais elle manifeste aussi envers le texte traduit une forme aristocratique de mépris (1989, 66-68). Une telle méfiance à l'égard de la traduction des œuvres littéraires est encore plus marquée dans le domaine franco-italien à cause de la primauté de la langue française dans la péninsule, primauté qui commence à s'affaiblir seulement vers la moitié des années $1930^{18}$. Mais cette position de Croce sera en partie modifiée entre 1939 et 1941 lorsque le philosophe, en invoquant l'autorité de Goethe, acceptera de nuancer ses propos sur l'impossibilité de la traduction littéraire ${ }^{19}$. Selon une thèse de Goethe commentée par Croce, on ne peut apprécier la qualité poétique d'une poésie que si on la réduit en prose $(1945,92)$. D'après le philosophe, on peut donc affirmer à la suite de Goethe que, paradoxalement, la traduction en prose d'un texte poétique est non seulement possible mais aussi souhaitable.

Gianfranco Contini met en évidence plusieurs contradictions fondamentales dans l'évolution de la pensée philosophique et esthétique de Croce, ce qui l'amène à énoncer la thèse de l'existence d'un premier, d'un deuxième, d'un troisième, voire d'un quatrième Croce $^{20}$. Mario Fubini souligne qu'au fil du temps la position traductologique du philosophe a évolué, en se faisant beaucoup plus complexe et nuancée: même dans l'Estetica, cette conception était d'après lui déjà ambivalente, donc pas dogmatique ${ }^{21}$. La vision de la traduction chez Croce en 1939-1941 se rapproche des thèses que

${ }^{18}$ Cf. J.-P. Viallet, « Statistiques et histoire... », 260.

19 B. Croce, "Intorno a un'antologia di traduzioni italiane delle liriche del Goethe » (1939), in Goethe (1919), Bari, Laterza, 1946, vol. II, 148-162 ; " Il giudizio della poesia su traduzioni » (1941), in Discorsi di varia filosofia, Bari, Laterza, 1945, vol. II, 90-94. D’après N. Mátyus («Il dibattito di Croce e Gentile... », 449), stimulé par ce débat avec Gentile, Croce serait à la recherche d'un critère esthétique capable de motiver l'autonomie de la traduction comme œuvre d'art mais aussi sa relative dépendance de l'œuvre originale.

20 G. Contini, La parte di Benedetto Croce nella cultura italiana, Turin, Einaudi, 1972, 30, 36 et 49 ; ce texte fut écrit en 1951 mais publié pour la première fois en 1966. La thèse de l'existence de plusieurs étapes dans la pensée de Croce est relayée aussi par G. Galasso, Croce e lo spirito..., chap. V et XV.

${ }^{21}$ M. Fubini, «Sulla traduzione » (1962), in Critica e poesia, Rome, Bonacci, 1973, 279-280, note 2. Selon N. Mátyus («Il dibattito di Croce e Gentile... », $445,446)$, pour Croce l'expression du texte-cible n'est jamais équivalente à celle du texte-source puisque le contenu de l'œuvre d'art a été aussi modifié par les sentiments qui ont guidé le travail du traducteur : le texte d'arrivée est donc autonome et différent. 
Debenedetti a forgées en 1925 sur la traduction de la musicalité proustienne $(1982,83)$. Maintenant, même pour Croce, dans la voix du traducteur doit résonner la voix du poète traduit et le ton du texte original doit se faire bien entendre dans la traduction; d'où ce sentiment de nostalgie du texte original que la traduction doit susciter et cultiver chez son lecteur $(1946,161)$. Croce souligne aussi que ce sentiment nostalgique dégagé par la traduction est déjà évoqué chez Goethe comme un désir irrésistible de l'original (162). Malgré cette ouverture, le philosophe ne manquera pas de se prononcer négativement, en 1941, sur la traduction de la musicalité proustienne : dans certains cas seulement, la traduction en prose peut conserver la puissance de l'inspiration, le rythme profond de l'original ; pour les œuvres de Mallarmé et des autres écrivains français décadents, y compris Proust, la traduction est impossible car la musicalité n'est que simple sonorité (1945, 92-93).

Si l'on prend alors en considération un jeune critique littéraire comme Debenedetti, qui débute en 1922 avec sa revue turinoise Primo tempo et qui se fait le chantre de Marcel Proust dès 1925, on constate que, pour se frayer un chemin original tout en gardant une attitude respectueuse vis-à-vis de Croce, il doit inscrire d'emblée sa réflexion traductologique dans une conception esthétique postcrocienne. Ceci est d'autant plus vrai que même son proustisme est incompatible avec la critique de Proust chez Croce: le déjà vu caractérisant les intermittences du cœur de la Recherche, phénomène dont Debenedetti s'occupe dès son premier essai sur Proust, est pour Croce inacceptable par principe car l'art est intuition, l'intuition individualité et l'individualité ne se répète pas, d'où l'impossibilité de revivre deux fois exactement la même expérience esthétique. La fonction de la mémoire dans la création artistique crocienne occupe du reste une place négligeable car l'intuition est d'après lui un processus intérieur prélogique et unique. De plus, comme sa conception historique de la réalité est très religieuse et morale, la reconstruction proustienne du passé constitue "la semplice soddisfazione edonistica di un piacere fisiologico », répondant d'après lui à une inquiétude sexuelle décadente (1945, 140-141). L'histoire, chez les lettrés français comme Proust, est pour Croce « una sorta di donnetta impudica, procuratrice di rare e squisite commozioni ai nervi ammalati » (145). Pour échapper à l'impasse totale de l'esthétique crocienne, Debenedetti choisira alors une démarche cohérente avec sa vision de la littérature comme dialogue ininterrompu entre générations, comme suprême expression de la tradition littéraire: si Croce, le «père intellectuel », n'est pas un exemple à suivre, il faut s'adresser à Francesco De Sanctis car ce 
dernier a nourri la critique littéraire de Croce (et de Gentile, du reste ${ }^{22}$ ). Le retour fondamental à De Sanctis chez Debenedetti naîtra donc de cette exigence de dépasser Croce sans s'y opposer ouvertement, de devenir « postcrociano » sans avoir été d'abord " anticrociano ». Son étude sur De Sanctis ne parait qu'en 1934 (1999, 381-401), mais la lecture de De Sanctis marquera sa découverte de Leopardi et des Operette morali, livre qu'il commence à lire en compagnie de Mario Fubini en 1923. Lorsqu'il s'exprime sur la traduction de la musicalité proustienne en 1925, Debenedetti a d'après nous déjà découvert avec admiration le De Sanctis critique de la traduction léopardienne. Mais il ne cite et commente pour la première fois le Studio su Giacomo Leopardi de De Sanctis que dans un essai de $1927^{23}$. Toutes les citations du critique napolitain que rapporte Debenedetti sont tirées du chapitre IV de cette étude, dans lequel De Sanctis analyse un recueil de traductions de Leopardi concernant l'œuvre du poète grec $\mathrm{Mosco}^{24}$. De Sanctis y fait l'éloge de Leopardi traducteur car, dans un élan d'empathie avec l'auteur traduit, il a su s'identifier totalement au personnage du poème; d'après lui, la voix de l'autre semble alors résonner dans l'âme du poète italien, comme s'il s'agissait de l'écho de sa propre voix intérieure. Pour Debenedetti, ces propos sont très représentatifs de l'approche critique de De Sanctis et il déclare que le critique napolitain offre dans ce chapitre un exemple de premier choix d'analyse de la traduction littéraire.

Par quels étranges cheminements, à deux époques distinctes et malgré leurs divergences esthétiques sur Proust, Croce et Debenedetti parviennent-ils à proposer la même définition de la traduction? On peut émettre l'hypothèse de l'existence de plusieurs sources à la base de cette conception commune: d'une part, l'héritage de la culture allemande qui marque de son empreinte l'idéalisme italien; d'autre part, l'influence décisive que De Sanctis exerce sur la formation de Croce dans la seconde moitié du XIXe siècle et sur celle du critique turinois dans les années 1920. Sur la relation complexe de Croce avec De Sanctis, Pierre Antonetti affirme :

Croce «sort » de De Sanctis, il s'en est nourri, il l'a intégré à son propre système, il s'est fait le paladin, le champion du vieux maître, et a fini par l'imposer à «l'intelligentsia » de son pays, non sans luttes ni polémiques $(1963,431)$.

\footnotetext{
${ }^{22}$ Le retour à De Sanctis est aussi un mot d'ordre de Gentile, relayé par une étude de Luigi Russo parue en 1928: Francesco De Sanctis e la cultura napoletana (cf. A. Gramsci, Letteratura e vita nazionale, 3-6, 17-18).

${ }_{23}$ Cf. G. Debenedetti, « Critica e autobiografia » (1927), in Saggi, 355-365.

24 F. De Sanctis, "Gli “idillii” di Mosco », in Leopardi (1885), Bari, Laterza, $1961,29-45$.
} 
Donc, d'après Antonetti, « revenir à De Sanctis c'est, en somme, débarrasser De Sanctis de l'interprétation de Croce et retrouver le maître à travers les extrapolations du disciple » $(1963,431)$. Or, De Sanctis a été très influencé par les idées d'Hegel et par les œuvres des frères Schlegel (220-236). Ces deux frères, critiques littéraires et traducteurs, ont fortement contribué à la réflexion sur la littérature et la traduction qui, à l'orée du XIXe siècle, a marqué la culture allemande ${ }^{25}$. De même, nous avons constaté que le désir du ton original comme visée de la traduction est d'abord une thèse de Debenedetti lecteur de De Sanctis en 1925, puis en 1939 de Croce lecteur de Goethe. De Sanctis se présente ainsi, dans la seconde moitié du XIXe siècle, grâce peut-être à son exil zurichois, comme l'intermédiaire privilégié entre cette culture allemande et la critique littéraire de l'Italie postunitaire. Le retour à De Sanctis permet alors à Debenedetti de s'ouvrir davantage à une composante fondamentale de la culture européenne que Croce, au nom de son esthétique, avait pendant plusieurs décennies négligée, sinon niée.

Debenedetti ne prend donc pas en considération les idées de Gentile sur l'art ou sur la traduction : nous pensons que cette prise de position est déterminée surtout par le choix politique de Gentile. Ce dernier, lorsqu'il est accusé par certains fascistes de ne pas être assez ou pas du tout fasciste, répond en disant que non seulement il est fasciste mais qu'il a été aussi l'un des précurseurs du fascisme ${ }^{26}$. Alors que, dans l'état actuel des débats, les avis sont très partagés sur «le degré de fascisme » à attribuer à Gentile ${ }^{27}$, il est évident que pour les antifascistes du Ventennio Gentile était bien fasciste ; lui-même y a cru jusqu'à sa mort $^{28}$. En 1920, Gentile n'est pas encore fasciste : il entre dans le gouvernement de Mussolini en 1922 comme ministre de

${ }^{25} \mathrm{Cf}$. à ce sujet A. Berman, L'épreuve de l'étranger, Paris, Gallimard, 1984, 2535 .

${ }^{26} \mathrm{Cf}$. A. Tarquini, Il Gentile dei fascisti. Gentiliani e antigentiliani nel regime fascista, Bologne, Il Mulino, 2009, 67 (où l'on cite la lettre de Gentile publiée dans La Tribuna en avril 1926).

${ }^{27}$ Alessandra Tarquini propose un panorama introductif, riche et complexe, de ce débat (2009, 11-22).

28 Après avoir lu le livre d'Alessandra Tarquini, on peut avancer cette réflexion : si des individus athées, catholiques cléricaux ou même juifs (avant 1938), monarchistes, républicains, traditionalistes ou révolutionnaires arrivent à se reconnaître dans le même groupe politique, quels principes fondamentaux partagent-ils ? Deux sans aucun doute : la foi dans la dictature et la violence pour imposer leur conception du monde; la haine de la démocratie et du dialogue respectueux des autres. Or, parmi ces fascistes, il y en a aussi qui se posent cette question quasi rhétorique : quelle est alors la différence entre les dictatures de Mussolini et de Staline? Au lieu d'alimenter des révisionnismes opportunistes, Il Gentile dei fascisti permet d'apprécier vraiment la valeur des principes fondateurs du dialogue social, du pacifisme et de la démocratie parlementaire. 
l'Éducation nationale, mais il n'est pas militant ni inscrit au parti, il n'a pas été «squadrista ». Néanmoins, comme il revendique son rôle de devancier, on pourrait se demander si sa théorie de la traduction n'est pas elle aussi fasciste ante litteram. Mais suffit-il d'utiliser cet adjectif pour marquer idéologiquement une pensée philosophique ou une pratique culturelle ? Giovanni Gentile est négligé en Italie dans certains ouvrages de traductologie ${ }^{29}$. Par exemple Umberto Eco, dans un chapitre de Dire quasi la stessa cosa consacré à la traduction herméneutique, cite M. Heidegger, H.G. Gadamer, G. Steiner, P. Ricœur mais jamais ce penseur qui les précède ${ }^{30}$. Nous insistons sur ce point parce que sa conception de la traduction, à la différence de celle de Croce, nous paraît remarquable. Le courant philosophique idéaliste de Gentile, l' «actualisme ", permet à ce penseur sicilien de donner en 1920 une interprétation de l'acte de la traduction très proche des positions théoriques de $\mathrm{W}$. Benjamin ${ }^{31}$. La convergence inattendue et peu explorée entre Gentile et Benjamin est certainement due à la matrice philosophique commune de l'idéalisme. Selon Charles Alunni,

la philosophie gentilienne - et plus généralement la dimension italienne de l'Historicisme - fut tout autre chose qu'un provincialisme «italique », et bien plutôt un moment essentiel de la philosophie européenne $(1988,9)$.

Le philosophe allemand Walter Benjamin écrit « La tâche du traducteur » en 1923, trois ans après l'intervention de Gentile sur ce sujet (quant à ce dernier, il reviendra sur la question en 1931 sans

29 Dans La teoria della traduzione nella storia, S. Nergaard (éd.), Milan, Bompiani, 1993, la première moitié du XXe siècle ne compte qu'Ortega y Gasset, Croce et Benjamin. Dans Teorie contemporanee della traduzione, S. Nergaard (éd.), Milan, Bompiani, 1995, le nom de Gentile n'est même pas cité dans la section philosophique. Il est en revanche cité dans une bibliographie raisonnée (D. D’Oria-M. Conenna, «Bibliografia critica sulla teoria della traduzione in Italia. 1920-1980 ", Il lettore di provincia, XII, $\mathrm{n}^{\circ} 44,1981,7-8$ ) mais on lui reproche entre autres d'avoir abordé en 1920, « in modo confuso », ce qu'en 1959, dans son célèbre essai « Aspects linguistiques de la traduction », R. Jakobson définira comme la traduction « intralinguale ou reformulation » (cf. Essais de linguistique générale, Paris, Minuit, 1963, 79).

${ }^{30}$ U. Eco, Dire quasi la stessa cosa, Milan, Bompiani, 2003 : il n'est pas cité non plus dans l'index des noms.

${ }^{31}$ Dans son introduction et dans les nombreuses notes qui accompagnent sa traduction de ce texte, Charles Alunni analyse les points de contact existant entre les deux philosophes: cf. G. Gentile, " Du tort et du droit des traductions ", Le Cahier du Collège international de philosophie, $\mathrm{n}^{\circ}$ 6, 1988, 13-20. 
modifier fondamentalement son discours) ${ }^{32}$. Deux points convergents chez Gentile et Benjamin sont à nos yeux dignes d'être analysés. D'une part, pour Benjamin la nécessité de la traduction est inscrite dans l'original lui-même qui contient toujours ses propres lois de traduction ; si dans sa survie - véritable «mutation et renouveau du vivant » (1971, 265) - cet original se modifie, c'est à la forme par excellence de la traduction qu'il revient de " mettre en lumière la post-maturation de la parole étrangère, les douleurs obstétricales de sa propre parole $» 33$ (266). D'autre part, pour Benjamin, le travail du traducteur s'oriente vers "l'insaisissable" qui se cache derrière le mystère poétique de l'œuvre; d'après lui, c'est « le pur langage » qui est exilé dans l'œuvre originale. Ce pur langage se manifeste « de façon intensive » dans la traduction (273). La tâche du traducteur est alors de racheter ce pur langage dans sa propre langue, de le libérer en le transposant, travail semblable à celui du philosophe car la nostalgie du pur langage qui s'annonce dans la traduction guide l'exégèse du traducteur et motive sa réécriture : la visée intentionnelle de la traduction est d'éveiller dans la langue d'arrivée l'écho du texte original. À la différence de la création littéraire, la traduction se tient hors de la forêt de la langue, "face à elle, et, sans y pénétrer, elle y fait résonner l'original, aux seuls endroits où, dans sa propre langue, elle peut faire entendre l'écho d'une œuvre écrite dans une langue étrangère » (269). Le pur langage se manifeste donc au premier chef dans les lieux textuels de la traduction où résonne l'écho de l'œuvre originale. Ainsi, à certains endroits privilégiés du texte traduit, se superposent l'ineffable poétique, le pur langage et l'écho de l'original: ce triplet représente pour Benjamin le mythe ultime de l'indicible.

La possibilité théorique de la traduction que prônent Debenedetti, Gentile et Benjamin dans les années 1920 est inscrite dans la nature même de l'acte de parole qu'ils considèrent comme l'expression d'une pensée universelle. Gentile conçoit la traduction

32 G. Gentile, « La critica e il tradurre », in La Filosofia dell'Arte (1931), Florence, Sansoni, 1937, 278-282 ; pour la traduction de ce texte et un bref commentaire, cf. R. Filippi, « La théorie de la traduction... », 54-57. W. Benjamin, " La tâche du traducteur », in Mythe et violence, Paris, Denöel, 1971, 261-275. Ces penseurs feront des choix politiques opposés mais leurs destins sont tous deux tragiques : Benjamin n'a pas encore lu Marx quand il écrit ce texte, il le découvre en 1924 et par la suite, étant juif et marxiste, il sera traqué par les nazis ; il se donnera la mort le 26 septembre 1940 à Portbou, dans la crainte d'être refoulé en France et de tomber ainsi aux mains de la Gestapo.

33 Sur le concept de forme chez Benjamin comme organisme textuel vivant régi par un principe d'organisation, à savoir la loi de « traduisibilité » inscrite dans l'original, cf. A. Berman, L'âge de la traduction : "La tâche du traducteur » de Walter Benjamin, un commentaire, Saint-Denis, Presses universitaires de Vincennes, 2008, 54-55. 
comme la démarche interprétative d'un sujet placé dans l'éternel mouvement de l'histoire et de la langue. Dans son incipit, il présente rapidement « le tort » des traductions, leur impossibilité conceptuelle, mais il n'adhère pas à cette position qu'il estime être "un lieu commun »34 (1988, 16). Il entre en revanche dans la question du «droit» en postulant une série de principes très chers à Henri Meschonnic : la forme littéraire n'est pas quelque chose qui s'ajoute au contenu ; ce dualisme d'origine aristotélicienne doit être décidément combattu car « la forme n'est autre que le contenu arraché à la staticité [...] et qui se trouve alors reconduit à cette vie réelle et concrète qui constitue sa génération interne »35(17)Le signe linguistique discontinu, statique, fini, laisse la place au discours continu et en mouvement, assumé par un sujet historique. Autrement dit, "la forme est la vie, c'est-à-dire la véritable réalité du contenu : sa subjectivité. L'art comme forme est cette subjectivité en acte qui se [replie] sur soi et n'exprime qu'elle-même » (16). La langue dans son caractère spirituel n'est donc pas pour Gentile « un fait » figé, ce contenu qu'il appelle « le parlé » et qui constitue l'objet de connaissance du linguiste et du grammairien, mais « un acte» vivant, la forme de la pensée du linguiste et du grammairien qu'il appelle «le parler»: comme dans le Cours de linguistique générale de Ferdinand De Saussure, paru en 1916, la langue («le parlé ») s'oppose ici à la parole («le parler»), et comme chez Émile Benveniste ou dans la pragmatique de John Austin (les speech acts ${ }^{36}$ ), l'énoncé s'oppose ici à l'énonciation, le produit grammaticalisé à l'acte de sa propre production. Gentile spécifie du reste que ce caractère spirituel de la langue doit être conçu, "selon la célèbre phrase de Humboldt, non pas comme ergon [travail] mais comme energeia [force en action] » (16). C'est la conception du langage chez Wilhelm von Humboldt, philosophe et linguiste allemand de la première moitié du XIXe siècle, qui relie donc étrangement Meschonnic à Gentile: le premier cite ce linguiste (y compris sa distinction ergon / energeia (Meschonnic 1999, 169) à maintes reprises dans ses ouvrages à partir des années 1970 et lui consacre même plusieurs travaux ${ }^{37}$. Par son caractère spirituel universel et vivant, la langue de Gentile est une forme unitaire, toujours la même langue (par rapport à

34 À la fin, Gentile parlera même de «préjugé » («Du tort et du droit des traductions », 20).

35 Cf. H. Meschonnic, Poétique du traduire, Lagrasse, Verdier, 1999: sur la dualité du signe, 115, 117, 142-143, 190 et passim ; sur l'activité du discours, par exemple, 12, 56, 97-111, 116, 168 sq.

${ }^{36}$ Ce philosophe de l'école d'Oxford tiendra ses conférences sur les actes de langage en 1955.

37 Cf. par exemple « Poétique d'un texte de philosophe et de ses traductions : Humboldt, Sur la tâche de l'écrivain de l'histoire " (Meschonnic 1999, 34339). Pour d'autres études de Meschonnic sur ce linguiste, cf. (1999, 343), note 1. 
la diversité des langues dans l'espace et dans le temps), mais toujours différente car vivante et en perpétuel développement; elle résonne inquiète dans l'âme humaine et se transforme, en changeant même entre deux instants consécutifs. D'où l'importance aux yeux de Gentile de l'acte de traduire qui est la condition de toute pensée comme de tout apprentissage puisque «l'on ne traduit pas seulement d'une langue étrangère dans la nôtre, [...] mais l'on traduit aussi à partir de la nôtre, toujours » (18). Le discours de Gentile se fait même borgesien là où il s'imagine en train de lire Dante en italien :

Je ne veux rien y mettre de moi. Je ne le commente pas, je ne remplace pas un mot par un autre, pas même, étant italien, pour formuler à la manière d'aujourd'hui ce que l'on formulait autrement au XIVe siècle [...]. Mais répéterais-je pour autant les mots qui furent ceux du poète? (19)

La répétition est impossible, même en gardant les mots de Dante car ces mots, dans leur réalité historique, vivent d'une vie qui éternellement se renouvelle : le philosophe anticipe ici le défi du Pierre Ménard traducteur du Quichotte ${ }^{8}$. Dans le premier chapitre de sa Filosofia dell'Arte, Gentile affirme du reste qu'aucun mot au plan sémantique n'est synonyme d'un autre mot ou de lui-même "poiché ogni parola ha quel significato in quel contesto, e il contesto è sempre un discorso che è un pensiero, in cui si oggettiva il soggetto $\gg 39$.

À l'instar de Benjamin, Gentile est également de l'avis que la traduction exprime le rapport le plus intime entre deux langues : elle les intègre en effet dans une "unique langue » où toutes les deux s'accordent et se complètent. Dans l'acte de la traduction, le rapport entre les deux langues atteint une unité linguistique supérieure :

Mais qu'est-ce que traduire, non pas in abstracto mais in concreto, lorsque quelqu'un traduit et que l'on prête attention à ce qu'il fait, si ce n'est interpréter ${ }^{\circ}$ ? Interprétation dans laquelle on passe d'une langue à l'autre parce qu'elles sont toutes les deux connues du traducteur, qu'il les a mises en rapport dans son esprit, passant de l'une à l'autre comme d'une partie à l'autre de la même langue: de cette unique langue qui existe véritablement pour lui : langue qui n'est ni l'une ni l'autre, mais l'ensemble des deux dans leur rapport et dans leur unité (18).

On peut considérer le pur langage de Benjamin comme une définition différente de ce même concept. Lorsque le traducteur accomplit le passage d'une langue à l'autre, selon Gentile il ne fait que

${ }^{8}$ Cf. J.L. Borges, « Pierre Ménard, auteur du Quichotte» (1941), in Fictions, Paris, Gallimard, 2003.

39 Cité par R. Raggiunti, «Il concetto del tradurre... », 443. C'est nous qui soulignons l’identité chez Gentile du discours et de la pensée. 
transformer sa pensée " en continuant à développer, clarifier, rendre toujours plus intérieur à soi et plus subjectif ce qu'il a commencé à penser » (19). Il s'agit d'abord d'un phénomène mental complexe, qui s'inscrit dans une certaine durée, où interprétation et traduction ne font qu'un dans la pensée du même individu. Le traduire est conçu non pas en tant que produit mais en tant qu'acte de production, comme un processus psycholinguistique in fieri : ce passage d'une langue à l'autre est selon Jean-René Ladmiral un "no-man's-langue " situé entre le déjà plus du message-source et le pas encore du message-cible ${ }^{40}$, un entre-deux qui, selon la théorie interprétative actuelle de la traduction, fait l'objet d'une déverbalisation ${ }^{41}$. L'écriture de la traduction toutefois n'est pas prise en compte par Gentile du fait de son identification de la pensée avec l'expression verbale. Pour lui langue et pensée coïncident : il n'y a pas de pensée hors de la langue. Il se trouve en vérité que les recherches neurologiques contemporaines nient ce postulat idéaliste ${ }^{42}$. Pour Gentile, le passage de la pensée interprétative d'une langue à l'autre par le biais de l'unique langue est une " procédure » longue et compliquée. Cette pensée est non seulement une pensée langagière, " un discorso che è un pensiero ", mais aussi une pensée hautement métalinguistique dont la réflexivité est l'expression de la conscience de soi de l'Esprit : cette pensée est capable de se penser en train de penser et même de décrire la forme de son acte 43 . Dans la mesure où toute lecture demande un effort de compréhension, donc d'interprétation de la parole de l'autre, l'interprétation d'un texte par le traducteur est comparable à l'interprétation d'un texte par le lecteur: toute interprétation est donc une traduction et vice versa ${ }^{44}$. Le processus de la compréhension, en langue étrangère ou en langue originale, vise impérativement selon ce philosophe idéaliste à la reconstruction et à la création d'un texte nouveau tel qu'il est pensé par son lecteur dans

$4^{\circ 0}$ J.-R. Ladmiral, « Le salto mortale de traduire : éléments culturels et psycholinguistiques de théorie de la traduction ", in La traduction littéraire, V. Agostini-Ouafi et A. R. Hermetet (dir.), Transalpina, $\mathrm{n}^{\circ}$ 9, 2006, 65-66.

${ }^{41}$ Cf. D. Seleskovitch, M. Lederer, Interpréter pour traduire, Paris, Didier Érudition (Traductologie ; 1), 1984.

42 Cf. J.-P. Changeux, L'homme de vérité, Paris, Odile Jacob, 2002, 206, où entre autres ce neurologue de renom cite l'ouvrage collectif Thought without Language, L. Weiskrantz (éd.), Oxford, Clarendon, 1988. Cf. aussi sur ce point C. Fabrizio, Idee linguistiche e pratica della lingua in Giovanni Gentile, Pise Rome, Fabrizio Serra Editore, 2008, 23.

43 Claudia Fabrizio (Idee linguistiche e pratica..., 30) souligne également cette réflexivité métalinguistique.

44 C'est la traduction " intralinguale » dont parle R. Jakobson (cf. " Aspects linguistiques de la traduction », 79). Sur la relation complexe entre traduction et interprétation, cf. U. Eco, Dire quasi la stessa cosa, 225-253 («Interpretare non è tradurre »). Pour cette identification, cf. aussi chez H.G. Gadamer, «Dall'ermeneutica all'ontologia », in Teorie contemporanee della traduzione, 347. 
l'acte de la lecture et «non pas en tant qu'il fut pensé dans l'acte de son écriture » (19). L'œuvre d'art est donc foncièrement traduisible et infiniment interprétable car, n'ayant pas « une existence finie, achevée, close », elle n'est pas «matériellement prisonnière du temps comme de l'espace » (20). Chaque lecteur réécrit son texte selon sa sensibilité puisque l'œuvre littéraire est toujours une œuvre ouverte. Les implications herméneutiques de ces affirmations sont très stimulantes si l'on pense à la réflexion des années 1970 sur la réception et sur le rôle actif du lecteur dans l'interprétation des œuvres chez Wolfang Iser, Umberto Eco ou Hans Robert Jauss. Benedetto Croce avait au fond raison de s'insurger contre cette vision de la lecture-interprétationtraduction sans bornes ni balises, ouverte à toute déconstruction : d'après lui, cette identification allait ébranler les bases mêmes de la critique littéraire et artistique traditionnelle car au lieu d'étudier la vérité - unique et objective - d'une œuvre poétique, la critique se consacrerait alors à l'étude de remaniements, réécritures ou variations poétiques sans critères précis et clairs (1951, 308-309).

Ce débat des années 1920 en Italie, grâce notamment à la réflexion de Gentile, place la traduction au centre de toute opération cognitive - en devançant ainsi l'étude de R. Jakobson de 1959 (1963, 81,84 ) - par la capacité que seul a le langage de réfléchir sur lui-même ; mais il ouvre aussi les portes aux courants herméneutiques des années 1960-1970, puis au déconstructionnisme des décennies suivantes. Favorable sur le plan politique à la dictature violente et totalitaire où l'État écrase l'individu, Gentile nous lègue $a$ contrario une conception de la traduction marquée par un cognitivisme humaniste et une esthétique transgressive et libertaire. Si Gramsci et Debenedetti, sur la question de la traduction, ont su être parfaitement cohérents avec euxmêmes, Croce et Gentile sont restés prisonniers de leurs contradictions politiques, philosophiques et esthétiques.

\section{Références bibliographiques}

Abbagnano, Nicola. Storia della filosofia, vol. V, Verso il pensiero contemporaneo: dallo spiritualismo all'esistenzialismo. Novare: Istituto Geografico De Agostini, 2006.

Alunni, Charles. «Giovanni Gentile - Martin Heidegger ». In : Le Cahier du Collège international de philosophie, $\mathrm{n}^{\circ}$ 6, $1988:$ 7-12.

Antonetti, Pierre. Francesco De Sanctis (1817-1883): son évolution intellectuelle, son esthétique et sa critique. Paris: Ophrys (Annales de la Faculté des Lettres d'Aix-en-Provence ; n. s. 38), 1963.

Benjamin, Walter. "La tâche du traducteur ». In : Mythe et violence. Paris : Denöel, 1971, 261-275.

Borghese, Lucia. "Tia Alene in bicicletta. Gramsci traduttore dal tedesco e teorico della traduzione ». In : Belfagor, XXXVI, 1981 / $6: 635-665$.

Croce, Benedetto. «Il giudizio della poesia su traduzioni » (1941). In : Discorsi di varia filosofia, vol. II. Bari : Laterza, 1945 : 90-94. 
Croce, Benedetto. « Un caso di storicismo decadentistico » (1943). In : Discorsi di varia filosofia, vol. II. Bari : Laterza, 1945 : 138-145.

Croce, Benedetto. «Intorno a un'antologia di traduzioni italiane delle liriche del Goethe » (1939). In : Goethe (1919), vol. II. Bari : Laterza, 1946 : 148-162. Croce, Benedetto. « Indivisibilità dell'espressione in modi o gradi e critica della rettorica » (1902). In : Estetica. Bari : Laterza, $1950:$ 75-82.

Croce, Benedetto. "Possibilità e impossibilità delle traduzioni ». In : Conversazioni critiche, vol. IV. Bari : Laterza, $1951:$ 308-309.

Debenedetti, Giacomo. "Probabile autobiografia di una generazione ». In : Saggi critici. Prima serie. Milan : Il Saggiatore, 1969 : 9-36.

Debenedetti, Giacomo. "Proust 1925 ». In : Rileggere Proust e altri saggi proustiani. Milan : Mondadori, $1982: 79-95$.

Debenedetti, Giacomo. " Commemorazione del De Sanctis » (1934). In : Saggi. (éd.) A. Berardinelli. Milan : Mondadori (I Meridiani), 1999 : 381-401.

Ferme, Valerio. Tradurre è tradire. La traduzione come sovversione culturale sotto il Fascismo. Ravenne : Longo, 2002.

Fertonani, Roberto. « A proposito del tradurre ». In : Il Ponte, XIII, 1957 : 250254.

Filippi, Raimondo. " La théorie de la traduction en Italie au XXe siècle (19001960)». In : Équivalences : revue de l'Institut supérieur des traducteurs et interprètes de Bruxelles, $\mathrm{n}^{\circ}$ 21, 1992 / 1-2 : 31-77.

Galasso, Giuseppe. Croce e lo spirito del suo tempo. Milan : Il Saggiatore, 1990.

Gentile, Giovanni. " Du tort et du droit des traductions ». In : Le Cahier du Collège international de philosophie, $\mathrm{n}^{\circ} 6,1988: 13^{-20}$.

Jakobson, Roman. « Aspects linguistiques de la traduction » (1959). In : Essais de linguistique générale. Paris : Minuit, 1963 : 78-86.

Meschonnic, Henri. Poétique du traduire. Lagrasse : Verdier, 1999.

Nagy, József. " La filosofia politica di Croce e di Gentile ». In : Benedetto Croce 50 anni dopo - Benedetto Croce 50 év után. (éd.) K. Fontanini, J. Kelemen, J.Takács. Budapest : Aquincum, 2004 : 79-92.

Vattimo, Gianni. " Croce tra hegelismo ed ermeneutica ». In : Benedetto Croce 50 anni dopo - Benedetto Croce 50 év után. (éd.) K. Fontanini, J. Kelemen, J.Takács. Budapest : Aquincum, 2004 : 111-113.

Vitiello, Pippo. «Il libro francese a Firenze e in Italia fra Otto e Novecento ». In : Paragone, XL, $\mathrm{n}^{\circ}$ 476, $1989:$ 55-72. 